\title{
SELECTION OF CSI-BASED PRECODING TECHNIQUES IN THE HF CHANNEL
}

\author{
J. López-Pérez ${ }^{\dagger}$, L. Melián-Gutiérrez ${ }^{\dagger}$, I. Pérez-Álvarez ${ }^{\dagger}$, S. Zazo*, I. Raos*, B. Pérez-Díaz ${ }^{\dagger}$ \\ ${ }^{\dagger}$ Instituto para el Desarrollo Tecnológico y la Innovación en Comunicaciones (IDeTIC), Universidad de Las Palmas de G.C. \\ (ULPGC), Campus de Tafira, 35017 Las Palmas, Spain, e-mail: jlopez@idetic.eu \\ * Universidad Politécnica de Madrid (UPM), Dpto. SSR, E.T.S. Ingenieros de Telecomunicación, Ciudad Universitaria, 28040 \\ Madrid, Spain, e-mail: santiago@gaps.ssr.upm.es
}

Keywords: Multi-carrier, OFDM, CSI, SVD, bit-loading.

\begin{abstract}
Multi-carrier modulations are widely employed in ionospheric communications to mitigate the adverse effects of the HF channel. In this paper we show how performance achieved by these modulations can be further increased by means of CSIbased precoding techniques in the context of our research on interactive digital voice communications. Depending on communication constraints and channel parameters, we will show which of the studied modulations and precoding techniques to select so that to maximise performance.
\end{abstract}

\section{Introduction}

Our research group has been working in HF communications for several years [5]. One of the areas we are deeply interested in is interactive digital voice communications, with inherently low delay requirements. Our current research bases communications on multi-carrier techniques, in order to minimise the adverse effects of the HF channel [6]. In the desire to improve performance without introducing delay, we have been studying several precoding techniques, amongst them the application of Channel State Information (CSI) to transmission [4]. This paper further expands our research in the application of CSI to the HF channel in this context of multi-carrier low delay communications.

\subsection{Multi-carrier modulations}

In order to cope with selective fading, nowadays HF communications make extensive use of multi-carrier modulations [8]. More than one carrier is used to transmit data, each sub-carrier being modulated to a lesser rate than would be necessary to transmit their joint information by means of a single-carrier modulation. Thus, channel bandwidth can be considered as flat for each sub-carrier.

The most extended amongst multi-carrier techniques is Orthogonal Frequency Division Multiplex (OFDM). However, deep nulls in the channel can attenuate one or more sub-carriers so much that information can no longer be recovered. A classical technique to mitigate this effect is channel coding, widely known as Coded OFDM (COFDM). Another way to avoid deep nulls effects is to apply spreading schemes over OFDM, resulting in OFDM Code Division Multiplex (OFDM-CDM) [1], which makes use of orthogonal codes to distribute the information to be transmitted over all or a subset of all the available OFDM sub-carriers. Multiple access interference is a spreading disadvantage, usually requiring Multiple User Detectors (MUD) at receiver.

\subsection{Precoding techniques based on Channel State Information}

Precoding at transmitter based on channel state can achieve channel capacity and/or improve Bit Error Rate (BER). Contrary to solely detection-based methods, CSI also requires transmitter-side processing. Two techniques will be used in this paper: bit-loading, based on waterfilling principles, and Singular Value Decomposition (SVD) of the equivalent channel matrix [4]. The former will be applied to OFDM, COFDM and OFDM-CDM, while the latter to OFDM-CDM with the spread symbols regarded as a kind of multiple access giving rise to a non-diagonal equivalent channel matrix.

In a bit-loading scheme based on channel state and available transmit power, transmitter decides how to individually modulate each sub-carrier, from a set of possible modulations, as well as how the available transmitting power is distributed amongst them [2]. On the other hand, channel matrix decomposition by means of SVD is a joint transmitterreceiver processing scheme that allows to diagonalise a nondiagonal channel matrix, whose application to HF OFDMCDM communications was studied in a previous work [4].

As a source of non-ideality, this paper will consider the delay in acquisition of the channel state by the transmitter. Transmitter receives an updated channel from receiver, and applies that same channel for bit-loading or SVD until a new one is received, regardless of the current state of the actual channel. For SVD, non-ideal CSI causes a non-diagonal equivalent channel matrix even after processing at reception. This effect can be palliated by a receiver-side compensation technique which can partially or completely correct it $[4,7]$.

\section{Objectives}

The main objective of this paper is to help decide which of the CSI techniques that our group is currently studying should be chosen for a given communication system, with the 
constraint that delay should be kept at minimum. This constraint prevents us from using interleavers.

A decision must be taken between maximising either the bits per second or the communication robustness. If the former is desired, in order to maximise the number of bits in each symbol no coding can be applied to OFDM or spreading gain to OFDM-CDM. However, if a robust communication, measured as BER, is regarded as more important than throughput, spreading gain can be considered for OFDMCDM, while channel coding for OFDM, that is, COFDM.

Firstly, ideal CSI will be studied and considered as an upper bound on performance. Then, for the selected modulations non-ideal CSI by means of delay will be introduced, as well as in the case of OFDM-CDM compensation of SVD mismatch. Finally, results with delay normalised by channel coherence time will be shown.

\subsection{Simulation parameters}

To carry out the simulations, a 60 sub-carriers OFDM signal, transmitted at a rate of 1/30 seconds per OFDM symbol has been implemented. A Minimum Mean Square Error (MMSE) detector has been used at receiver. In the case of OFDMCDM transmissions a load of $2 / 3$ has been chosen, in order to achieve spreading gain. Thus, 40 symbols are spread over the 60 available sub-carriers, with an MMSE plus MUD detector receiver when SVD is not applied. For COFDM, a 2/3 convolutional encoder with a Viterbi decoder has been implemented, also using a MMSE detector.

Two different sets of sub-carrier modulations will be used: low and high density. Low density modulations consist on a subset of $n$ PSK, from BPSK to 16-PSK, while high density modulations correspond to a joint subset of both $n$ PSK and nQAM: BPSK, QPSK, 8-PSK, 16-QAM, 32-QAM and 64QAM. When OFDM and OFDM-CDM without spreading gain are selected, a constant bit rate per sub-carrier is used: QPSK for the low density case for a total of 120 bits per OFDM symbol, and 16-QAM for the high density one for a total of 240 bits. If COFDM and OFDM-CDM with spreading gain are selected, the payload is reduced by $2 / 3$, thus 80 and 160 bits per OFDM symbol are carried when using low and high density modulations, respectively. In case of applying the bit-loading algorithm, each sub-carrier will be assigned a different modulation (or even left unmodulated) with the constraint to preserve the same overall rate than before.

Three different delays have been considered corresponding to 33,66 and $100 \%$ of each channel coherence time.

\subsection{HF channel model}

For the present simulations, ITU-R recommendations for HF simulations have been selected [3]. Specifically, fast, moderate and slow channels have been studied. Table 1 shows the parameters defined for each channel. A noise bandwidth of $4.8 \mathrm{kHz}$ has been used for all simulations.

\begin{tabular}{|l|c|c|c|}
\hline Channel & $\begin{array}{c}\text { Time spread } \\
(\mathrm{ms})\end{array}$ & $\begin{array}{c}\text { Doppler } \\
\text { spread }(\mathrm{Hz})\end{array}$ & $\begin{array}{c}\text { Number of } \\
\text { paths }\end{array}$ \\
\hline Fast & 2 & 1 & 2 \\
Moderate & 1 & 0.5 & 2 \\
Slow & 0.5 & 0.1 & 2 \\
\hline
\end{tabular}

Table 1: ITU-R channel parameters.

Although all the previous channels have been studied, in this paper only results for the moderate channel are shown due to space constraints. Nevertheless, the overall conclusions that will be drawn for each multi-carrier and precoding technique have been found to be valid for all channels.

\section{Ideal Channel State Information}

When deep nulls are present in the signal bandwidth, the information assigned to affected carrier or carriers in an OFDM modulation can be inevitably lost. Moreover, this could give rise to a lower bound in achievable BER due to fading caused by multipath. Under these conditions, OFDMCDM outperforms OFDM even without spreading gain: spreading the information amongst all carriers ensure that only part of each information bit is affected, and so it may be possible to recover all information. Results without spreading gain and with low density modulations for the moderate channel are shown in Figure 1.

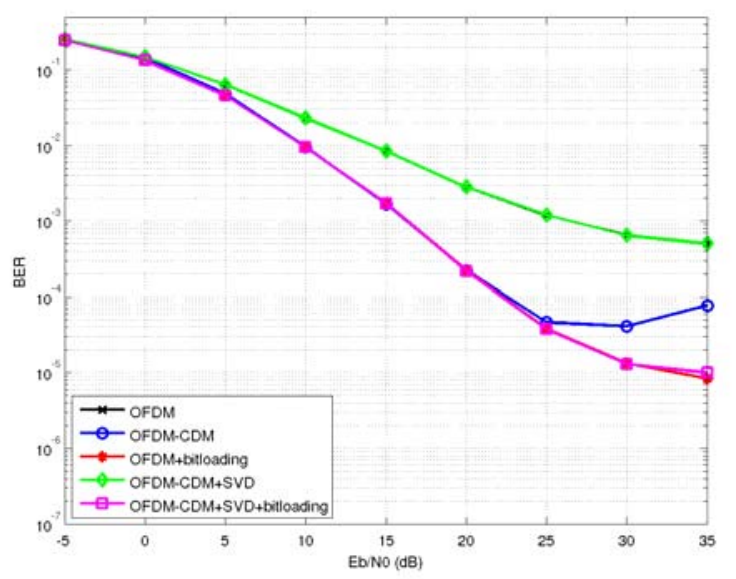

Figure 1: Ideal CSI, no spreading gain, low density modulations, moderate channel.

If bit-loading with perfect channel knowledge is applied over OFDM BER improves, as the bit-loading algorithm avoids those carriers most affected by the channel. If SVD decomposition is applied to OFDM-CDM an equivalent diagonal channel matrix is achieved, which is equivalent to an OFDM communication. Furthermore, it is expected that if bitloading is also applied on top of SVD results should be equivalent to OFDM, as Figure 1 shows. In other words, OFDM plus bit-loading and OFDM-CDM without spreading gain plus SVD plus bit-loading achieve the same performance. Thus, due to simplicity, OFDM plus bit-loading should be selected as the appropriate technique, or OFDM$\mathrm{CDM}$ if only low to medium $\mathrm{E}_{\mathrm{b}} / \mathrm{N}_{0}$ values are expected. 
If high density modulations are selected, results are shown in Figure 2. Although high density modulations allow for higher throughput, the highest density constellations are unsuitable for the HF channel, and thus bit-loading over OFDM or OFDM-CDM plus SVD achieves the worst results. In this case OFDM-CDM, with or without SVD, presents the best performance. Thus, as the simplest technique of the two, OFDM-CDM should be selected.

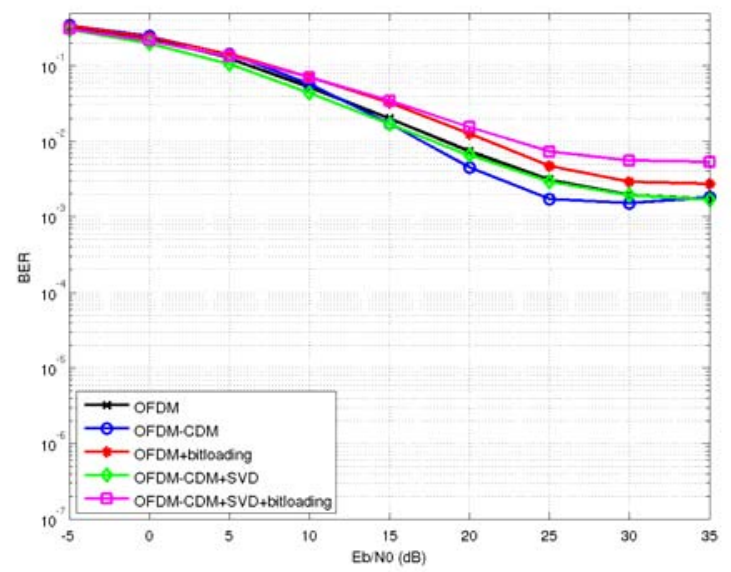

Figure 2: Ideal CSI, no spreading gain, high density modulations, moderate channel.

Results for OFDM-CDM with spreading gain and COFDM with low density modulations are shown in Figure 3 for a moderate channel. Although COFDM helps avoiding nonrecoverable loss of information due to deep nulls in the channel, the lack of interleaver makes it perform worse than OFDM-CDM with spreading gain. Nevertheless, by applying bit-loading we can increase performance without introducing the delay associated to interleavers. Once more, SVD is shown to have an equivalent performance to OFDM, and thus to increase performance bit-loading must be applied. The best overall performance is achieved by OFDM-CDM plus SVD plus bit-loading. However, for low to medium $\mathrm{E}_{\mathrm{b}} / \mathrm{N}_{0}$ values OFDM-CDM performance is comparable.

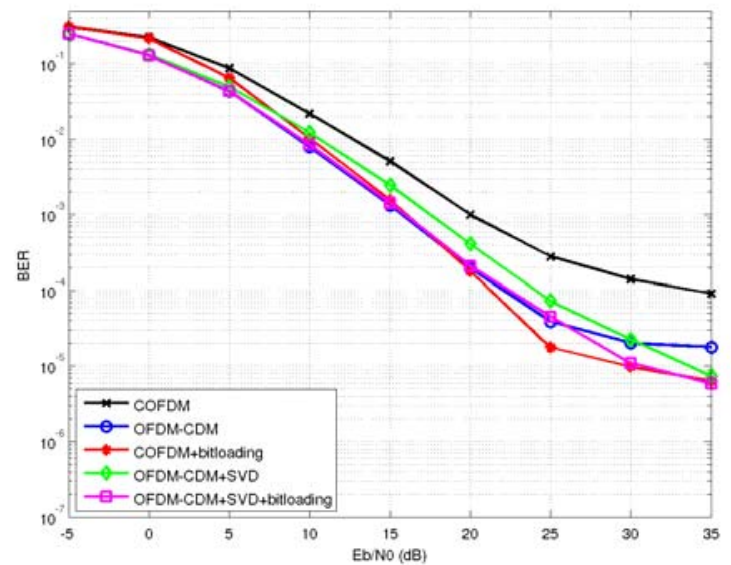

Figure 3: Ideal CSI, spreading gain, low density modulations, moderate channel.
For the case of high density modulations with COFDM and OFDM-CDM with spreading gain, results are shown in Figure 4. Once more, bit-loading with high density modulations shows itself unsuitable for the HF channel, with worse performance than with constant modulation per subcarrier. However, in this case OFDM-CDM with SVD outperforms OFDM-CDM, and so it should be the selected technique.

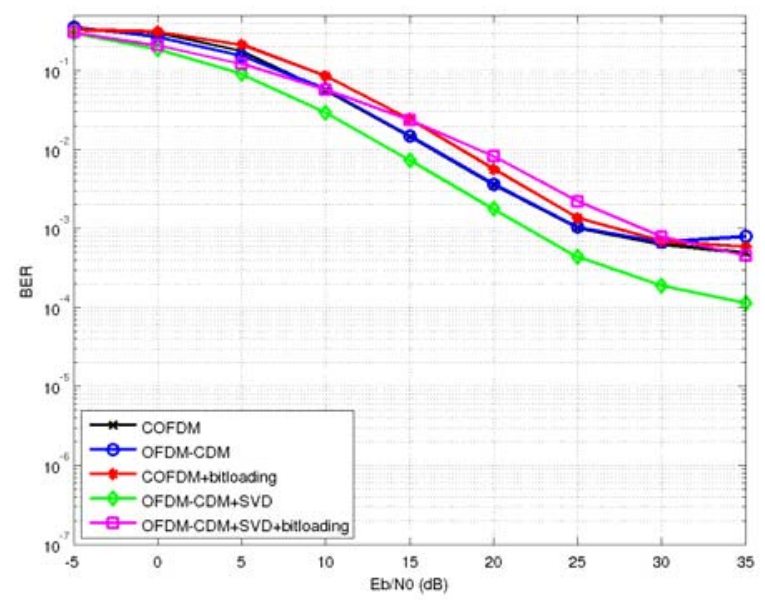

Figure 4: Ideal CSI, spreading gain, high density modulations, moderate channel.

\section{Non-ideal Channel State Information}

The effect of delay $(\Delta)$ in channel acquisition by transmitter is a worsening in BER achievable due to application of the wrong channel in bit-loading and SVD algorithms. The severity of this worsening depends on the length of the delay and the channel behaviour, mainly its coherence time.

As aforementioned, it has been shown in previous works that it is possible to compensate SVD decomposition mismatch between actual and applied channels in order to try to properly obtain a diagonal equivalent channel matrix, and thus partially or completely restore performance [4]. We will only show results with this receiver-side SVD compensation technique applied, which we will call SVD2 in the figures.

In the previous section we identified OFDM plus bit-loading, and OFDM-CDM for low to medium $\mathrm{E}_{\mathrm{b}} / \mathrm{N}_{0}$ values, as the contending techniques for low density modulations if no spreading gain is applied. The effects on performance of delay in channel acquisition can be seen in Figure 5. As delay increases, performance decreases gradually. Under these conditions, OFDM-CDM shows better performance for all simulated $\mathrm{E}_{\mathrm{b}} / \mathrm{N}_{0}$ values.

Similarly, Figure 6 shows results for the same low density modulations in the case of applying spreading gain to OFDMCDM. In this case, OFDM-CDM and OFDM-CDM plus SVD plus bit-loading were the contending techniques. Again, as delay increases, performance decreases, although in this case less dramatically than in the previous one. Once more, 
OFDM-CDM shows the best performance for all simulated points when the CSI technique faces delay.

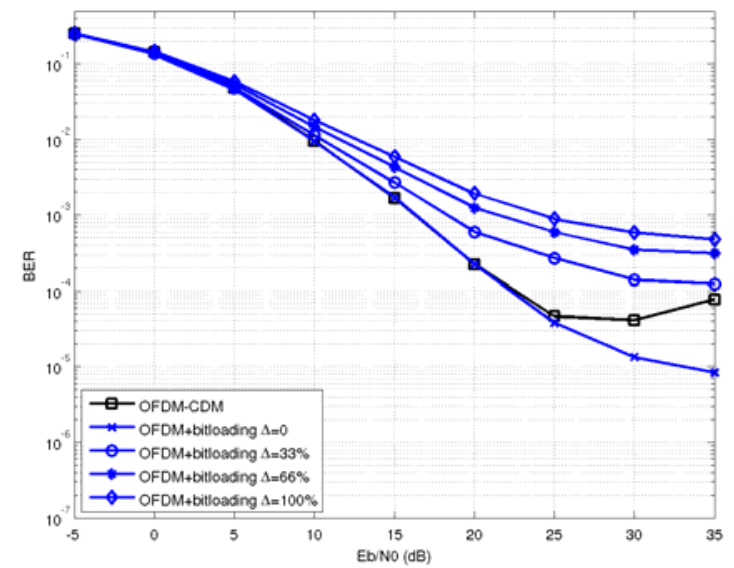

Figure 5: Non-ideal CSI, no spreading gain, low density modulations, moderate channel.

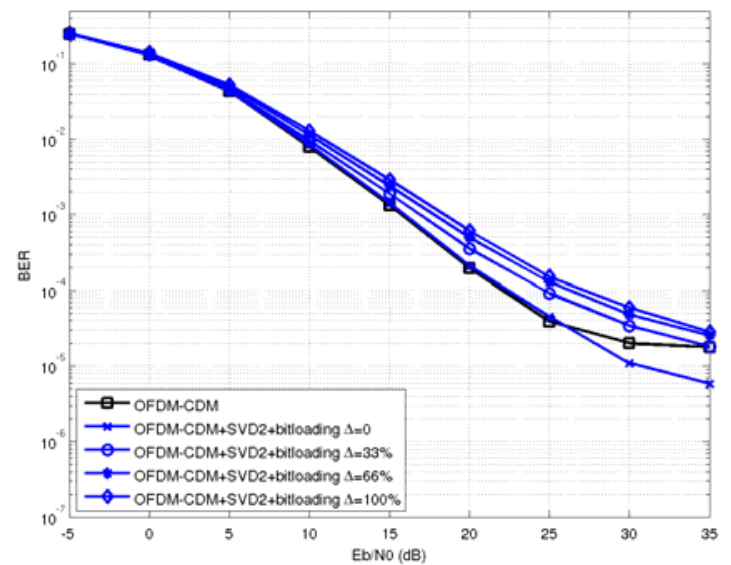

Figure 6: Non-ideal CSI, spreading gain, low density modulations, moderate channel.

Figure 7 shows results when spreading gain is applied for OFDM-CDM plus compensated SVD for high density modulations. Unlike with low density modulations, in this case performance of SVD due to non-ideal CSI is almost completely restored by the compensation technique. Thus, due to the damaging effects of the HF channel on the densest constellations, OFDM-CDM plus compensated SVD outperforms OFDM-CDM for all studied delays.

\section{Normalisation by channel coherence time for non-ideal CSI}

In order to try to abstract results from the type of channel in the case of non-ideal CSI, the delay in the application of the channel at transmitter has been normalised by the channel coherence time. Once more, only the previously selected algorithms are shown. In all figures, OFDM-CDM without CSI is shown as reference with dashed lines, as it has no dependency on delay.

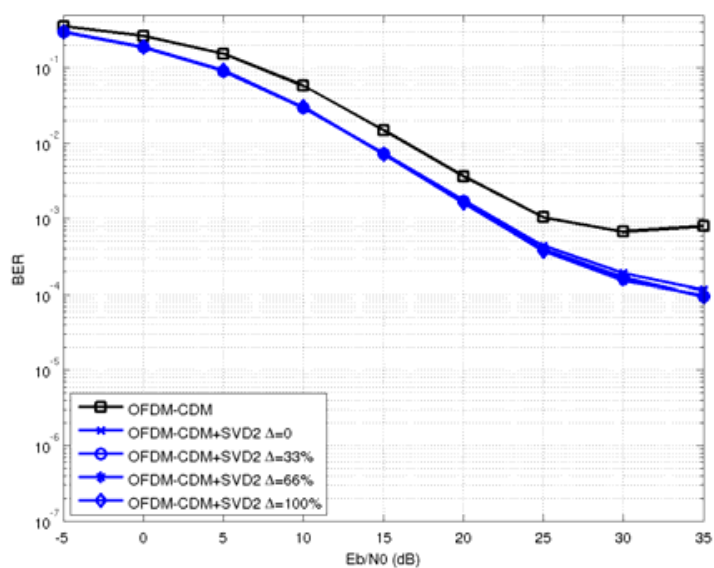

Figure 7: Non-ideal CSI, spreading gain, high density modulations, moderate channel.

Figure 8, Figure 9 and Figure 10 show performance when delay in acquisition of channel by the transmitter is normalised by the channel coherence time. A subset of five different $\mathrm{E}_{\mathrm{b}} / \mathrm{N}_{0}$ values is depicted.

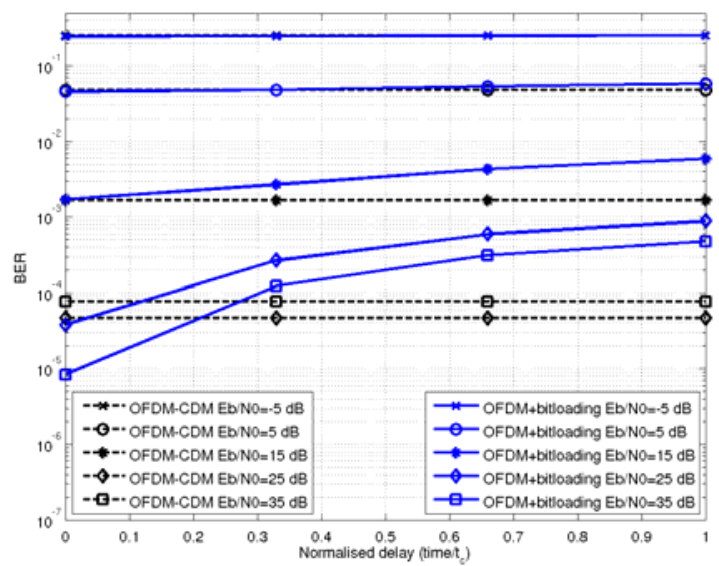

Figure 8: Normalised delay, no spreading gain, low density modulations, moderate channel.

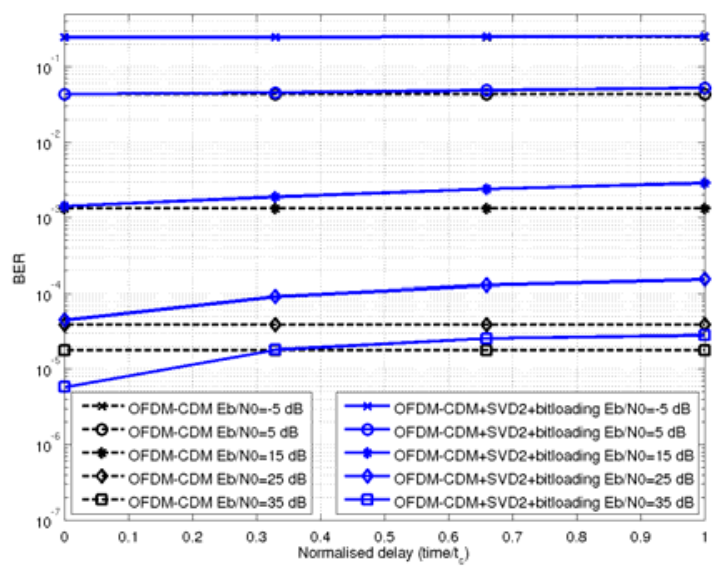

Figure 9: Normalised delay, spreading gain, low density modulations, moderate channel. 


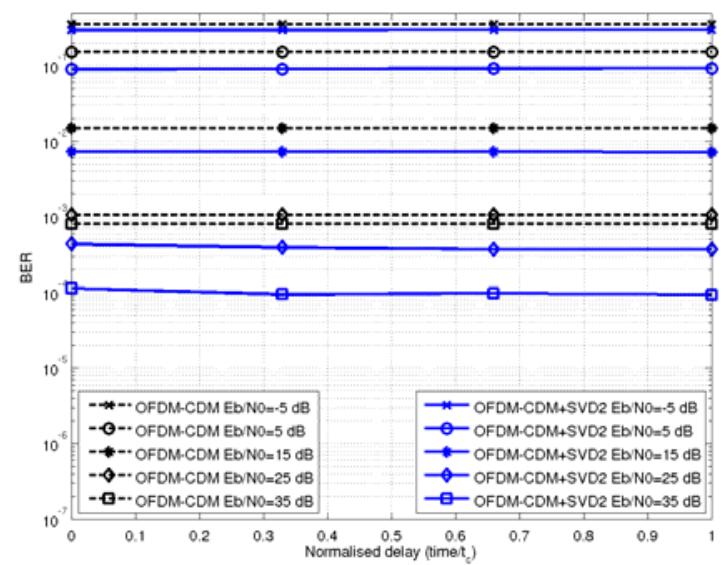

Figure 10: Normalised delay, spreading gain, high density modulations, moderate channel.

By using these types of figures with the delay normalised by the channel coherence time, if the expected range of $\mathrm{E}_{\mathrm{b}} / \mathrm{N}_{0}$ values that the communication system is likely to find and the maximum delay on channel acquisition by the transmitter are known, it is easier to select the most appropriate technique in order to achieve a target BER. In Figure 10 it can be seen that the non-ideal CSI does not affect performance, as curves are horizontal. In this particular case this is due to the receiverside correction technique to channel mismatch in SVD, which can completely restore performance.

\section{Conclusions}

If the objective is to maximise bit rate while using low density modulations for ideal CSI, then OFDM plus bitloading should be selected as its overall performance for all simulated $\mathrm{E}_{\mathrm{b}} / \mathrm{N}_{0}$ values is the best in these conditions. However, once delay is introduced in the CSI techniques, OFDM-CDM on its own can give better results from a certain delay onwards. Figures with delay normalised by the channel coherence time can help decide when to use one or the other, depending on the expected maximum delay and $\mathrm{E}_{\mathrm{b}} / \mathrm{N}_{0}$ range.

On the other hand, if the desire is to get a robust communication at the expense of throughput and so spreading gain is applied, for ideal CSI OFDCM-CDM plus receiverside correction to SVD for channel mismatch plus bit-loading is to be selected. However, if delay in CSI is introduced, figures with delay normalised can help decide up to which delay this technique outperforms OFDM-CDM for the expected range of $\mathrm{E}_{\mathrm{b}} / \mathrm{N}_{0}$.

If higher throughput is desired by using high density modulations, performance is severely penalised by the nature of the HF channel. Without spreading gain, the application of dense constellations to CSI techniques drastically decreases performance, and thus OFDM-CDM even without spreading gain outperforms all others.
However, if spreading gain is used in order to get a more robust communication, although with lower throughput, SVD with receiver-side correction for channel mismatch achieves the best performance. Due to the use of a constant modulation per sub-carrier with a less dense constellation than those use in some of the sub-carriers when bit-loading is applied, the application of this last one on top of SVD decreases performance. Furthermore, in this particular case the correction algorithm to the channel mismatch for SVD is capable of completely restoring performance. Thus, this technique should always be selected instead of the contending OFDM-CDM for any delay up to the channel coherence time.

\section{Acknowledgements}

This work was supported in part by the Spanish Ministry of Science and Innovation under grants TEC2010-21217-C02 CR4HFDVL, and CONSOLIDER-INGENIO CSD200800010 COMONSENS.

\section{References}

[1] K. Fazel and S. Kaiser, Multi-Carrier and Spread Spectrum Systems. New York: John Wiley and Sons, (2003).

[2] R. Fisher and J. Huber, "A new loading algorithm for discrete multitone transmission". Proceeding - Global Telecommunications Conference (GLOBECOM), 1, pp. 724-728, (1996).

[3] ITU-R F.1487. "Testing of HF modems with bandwidths of up to about $12 \mathrm{kHz}$ using ionospheric channel simulators". ITU-R Technical Report, (2000).

[4] J. López-Pérez, I. Pérez-Álvarez, S. Zazo-Bello, L.B. Melián-Gutiérrez and A. Amaro-García, "Impact of CSI latency on HF multi-carrier communications for delaysensitive applications", IRST 2009 - 11th IET International Conference on Ionospheric Radio Systems and Techniques, Edinburgh, UK (2009).

[5] I.A. Pérez-Álvarez, S. Zazo, M. Ghogho, J. López,. Experimental Results on Multicarrier MIMO HF Communications", 2011 URSI General Assembly and Scientific Symposium (GASS), Istanbul, Turkey (2011).

[6] H. Santana-Sosa, I. Pérez-Álvarez, S. Zazo-Bello, E. Mendieta-Otero, I. Raos and J. López-Pérez, "Performance over a real link of a HF software radio modem for interactive digital voice communications", IRST 2006 - 10th IET International Conference on Ionospheric Radio Systems and Techniques, London, UK (2006).

[7] M. Tesanovic, D. Bull, A. Doufexi, V. Sgardoni and A. Nix. "Impact of CSI latency on video quality in MIMO systems employing singular value decomposition". IEEE Electronic Letters, 43 (18), pp. 972-973, (2007).

[8] S. Zazo-Bello and E. Esteban. "Comparison of single carrier and multicarrier techniques for the HFDL system". Sixth Meeting of the Aeronautical Mobile Communication Panel (AMCP), Montreal, (1999). 